Preprint DFPD 95/TH/16

hep-th/

March, 1995

\title{
Note on manifest Lorentz and general coordinate invariance in duality symmetric models $\square^{*}$
}

\author{
Paolo Pasti，Dmitrij Sorokin [ and Mario Tonin \\ Università Degli Studi Di Padova Dipartimento Di Fisica "Galileo Galilei" \\ ed INFN, Sezione Di Padova Via F. Marzolo, 8, 35131 Padova, Italia
}

\begin{abstract}
We consider a generalization of a duality symmetric model proposed by Schwarz and Sen [1]. It is based on enlarging the model with a dynamical vector field being a time-like component of a local Lorentz frame. This allows one to preserve the manifest Lorentz invariance of the model in flat space-time. The presence of this field is regarded as a relic of gravitational interaction which respects the general coordinate invariance in curved space-time but breaks the local Lorentz symmetry in tangent space down to its spacial subgroup.

PACS: 11.15-q, 11.17+y
\end{abstract}

Key words: electric-magnetic duality, Lorentz symmetry, supersymmetry, general coordinate invariance.

*This work was carried out as part of the European Community Programme "Gauge Theories, Applied Supersymmetry and Quantum Gravity" under contract SC1-CT92-D789, and supported in part by M.P.I.

†on leave from Kharkov Institute of Physics and Technology, Kharkov, 310108, Ukraine.

e-mail: sorokin@pd.infn.it

${ }^{\ddagger}$--mail: tonin@pd.infn.it 
Following the course of studying duality in string theory and related topics Schwarz and Sen [1] proposed a class of gauge invariant actions which are manifestly invariant under duality transformations. "The price for doing this is the sacrifice of manifest Lorentz invariance or general coordinate invariance, though both symmetries can be realized nonetheless". But as it has already happened not one time, models, which at first have been considered as manifestly Lorentz non-invariant later on were reformulated in a Lorentz invariant way. The Green-Schwarz superstring is one of these examples [3].

In a recent paper [2] Khoudeir and Pantoja proposed a covariant form of the SchwarzSen action by introducing into the theory an auxiliary time-like constant vector. However, being a constant, this vector, in fact, violates Lorentz invariance. Hence, in spite of the nice covariant form of the action and equations of motion Lorentz invariance have not been restored.

Below we propose a generalization of the duality symmetric action [1, 2] by considering the auxiliary vector as a Lorentz frame vector field whose presence in the model can be regarded as a relic of a gravitational interaction. At the classical level this vector field completely decouples from the gauge field sector. We will see that the coupling of duality symmetric fields to gravity respects the general coordinate invariance in curved space-time but breaks the local Lorentz symmetry in tangent space down to its spacial subgroup.

For simplicity we will deal with the dual symmetric version of (supersymmetric) Maxwell theory, though the construction is generalized to the case of antisymmetric gauge fields [1] as well.

The duality symmetric form of the $\mathrm{D}=4$ Maxwell action proposed by Schwarz and Sen involves two abelian gauge fields $A_{m}^{\alpha}(\alpha=1,2 ; \mathrm{m}=0,1,2,3)$ which are present in the action on an equal footing:

$$
S=-\frac{1}{2} \int d^{4} x\left(B^{i \alpha} \mathcal{L}^{\alpha \beta} E_{i}^{\beta}+B^{i \alpha} B_{i}^{\alpha}\right)
$$

where

$$
E_{i}^{\alpha}=F_{0 i}^{\alpha}=\partial_{0} A_{i}^{\alpha}-\partial_{i} A_{0}^{\alpha}, \quad B^{i \alpha}=\frac{1}{2} \varepsilon^{i j k} F_{j k}^{\alpha}=\varepsilon^{i j k} \partial_{j} A_{k}^{\alpha},
$$

$i, j, k=1,2,3$ are spacial indices, and $\mathcal{L}^{\alpha \beta}$ is the antisymmetric unit matrix $\left(\mathcal{L}^{12}=1\right)$.

Apart from the ordinary abelian gauge transformations of $A_{m}^{\alpha}$ the action is invariant under local transformations

$$
A_{0}^{\alpha} \rightarrow A_{0}^{\alpha}+\Psi^{\alpha}(x)
$$

and global $S O(2)$ transformations which mix $A_{m}^{1}$ and $A_{m}^{2}$, its discrete subgroup being the duality symmetry:

$$
A_{m}^{\alpha} \rightarrow \mathcal{L}^{\alpha \beta} A_{m}^{\beta}
$$

Using the local symmetries and the $A_{m}^{\alpha}$ equations of motion one can eliminate one of the gauge fields and get the conventional Maxwell theory for the other one. Then the duality symmetry is reduced to the duality between the electric and magnetic strength of the remaining Maxwell field [1]. 
The action is also invariant under the spacial rotations, and has a global symmetry which is reduced to the Lorentz transformations when the model is reduced to Maxwell theory [1].

In the paper [2] by use of a constant time-like vector $u^{m}$ subject to the condition

$$
u^{m} u_{m}=-1
$$

the action (11) was rewritten in the following form

$$
S=-\frac{1}{2} \int d^{4} x u^{m} F_{m n}^{* \alpha}\left(\mathcal{L}^{\alpha \beta} F^{\beta n p}-F^{* \alpha n p}\right) u_{p}
$$

where $F_{m n}^{* \alpha}=\frac{1}{2} \epsilon_{m n p q} F^{\alpha p q}$ is the dual of $F_{m n}^{\alpha}=\partial_{m} A_{n}^{\alpha}-\partial_{n} A_{m}^{\alpha}$.

In (6) the transformations (3) take the form

$$
A_{m}^{\alpha} \rightarrow A_{m}^{\alpha}+u_{m} \Psi^{\alpha}(x)
$$

Since $u_{m}$ is a constant which satisfies (5) the action (6) corresponds to a different choice of the Lorentz frame in space-time and is reduced to (1) by an appropriate Lorentz transformation of $u_{m}$ to $u_{m}=-\delta_{m}^{0}$. Hence, in spite of the Lorentz covariant form, eq. (6) does not possess manifest Lorentz symmetry.

To get a Lorentz symmetric action one must consider $u_{m}$ as an $x$-dependent vector field and take into account its equations of motion in the presence of the orthonormality condition (5). Then $u_{m}$ becomes a component of a local frame $\left(u_{m}, u_{m}^{i}\right)$ in the flat spacetime, or a harmonic [4] of the Lorentz group, where $\left(u_{m}, u_{m}^{i}\right)$ satisfy the orthonormality condition (5) and

$$
u_{m} u^{i m}=0, \quad u_{m}^{i} u^{j m}=\delta^{i j} .
$$

Implicit use of (8) below will substantially simplify finding the symmetries and solving for the equations of motion of the model. fl In conclusion we will make a conceptual comment on the possibility of treating of $\left(u_{m}, u_{m}^{i}\right)$ as a relic of a gravitational field vierbein.

If $u_{m}$ is a field, the action (6) looses the local invariance (7) (or (3)), while the latter plays an important role in establishing the relationship of the model with Maxwell theory. Thus, eq. (6) must be modified in a way which restores this symmetry.

We write down the generalized action in the following form:

$$
S=\int d^{4} x\left(-\frac{1}{8} F_{m n}^{\alpha} F^{\alpha m n}+\frac{1}{4} u^{m} \mathcal{F}_{m n}^{\alpha} \mathcal{F}^{\alpha n p} u_{p}-\epsilon^{m n p q} \Lambda_{m n} \partial_{p} u_{q}+\lambda(x)\left(u_{m} u^{m}+1\right)\right) .
$$

For constant $u_{m}$ eq. (9) reduces to (6). From the form (9) of the action one may see where the duality symmetric model differs from the ordinary theory of two independent Maxwell

\footnotetext{
${ }^{4}$ Different types of the harmonic fields have been successfully used to solve covariance problems in various theories including superstrings. Due to the great amount of literature on this subject we will abstain from giving references.
} 
fields. The first term in (9) is just the sum of the two Maxwell terms. The nontrivial "interaction" between the two gauge fields is described by the second term, where

$$
\mathcal{F}_{m n}^{\alpha}=\mathcal{L}^{\alpha \beta} F_{m n}^{\beta}-F_{m n}^{* \alpha}, \quad \mathcal{F}_{m n}^{\alpha} \equiv \frac{1}{2} \epsilon_{m n p q} \mathcal{L}^{\alpha \beta} \mathcal{F}^{\beta p q}
$$

is the self-dual tensor. Note that because of this self-duality $\mathcal{F}_{m n}^{\alpha} \mathcal{F}^{\alpha n m}=0$ identically, which causes the problem of Lorentz covariant incorporation of (10) into the action.

The third term (where $\Lambda_{m n}(x)$ is an auxiliary antisymmetric gauge field) ensures the invariance of the action with respect to (7) provided $\Lambda_{m n}$ transforms as follows:

$$
\Lambda_{m n} \rightarrow \Lambda_{m n}+\Psi^{\alpha} \mathcal{F}_{m p}^{\alpha} u^{p} u_{n}-\Psi^{\alpha} \mathcal{F}_{n p}^{\alpha} u^{p} u_{m}
$$

One more, obvious, local symmetry of the action is its invariance (up to a total derivative) under the following transformations of $\Lambda_{m n}$ :

$$
\delta \Lambda_{m n}=\partial_{m} \Lambda_{n}(x)-\partial_{n} \Lambda_{m}(x) .
$$

The fourth term, where $\lambda(x)$ is a Lagrange multiplier, ensures (5).

The equations of motion obtained from the action (9) have the following form:

$$
\begin{gathered}
\delta A_{l}^{\alpha}: \quad \epsilon^{l m n p} \partial_{m}\left(u_{n} \mathcal{F}_{p r}^{\alpha} u^{r}\right)=0, \\
\delta \Lambda_{l m}: \quad \epsilon^{l m n p} \partial_{n} u_{p}=0, \\
\delta u_{m}: \quad-\frac{1}{2} \mathcal{F}_{n}^{\alpha m} \mathcal{F}^{\alpha n p} u_{p}+\epsilon^{m n l p} \partial_{n} \Lambda_{l p}=\lambda(x) u^{m} .
\end{gathered}
$$

In view of (14) the general solution to (13) is

$$
\mathcal{F}_{p r}^{\alpha} u^{r}=\partial_{p} \tilde{\Psi}^{\alpha}+u^{r} \partial_{r}\left(u_{p} \tilde{\Psi}^{\alpha}\right)
$$

where $\tilde{\Psi}^{\alpha}$ are arbitrary functions. But (again due to (14)) the transformation of $\mathcal{F}_{p r}^{\alpha} u^{r}$ with respect to (1) has the same form as (16). So, by putting $\Psi^{\alpha}=\mathcal{L}^{\alpha \beta} \tilde{\Psi}^{\beta}$ we can choose the gauge of (四) in which $\mathcal{F}_{p r}^{\alpha} u^{r}=0$ and, hence, $\mathcal{F}_{p r}^{\alpha}=0$ since it is self-dual. As a result we arrive at the duality condition obtained in [1, 2]:

$$
\mathcal{L}^{\alpha \beta} F_{m n}^{\beta}=\frac{1}{2} \epsilon_{m n p q} F^{\alpha p q}
$$

When (17) is satisfied, the first term in the l.h.s. of (15) is zero. Thus, on the mass shell the gauge field sector completely decouples from $u_{m}$ and $\Lambda_{m n}$, and, in compliance with [1], the former is reduced to ordinary Maxwell theory.

From (14) we get

$$
u_{m}=\partial_{m} \phi(x)
$$

(where (18) must satisfy (5)), while eq. (15) reads that (in the gauge (17)) the only non-zero component of the strength vector $\epsilon^{m n l p} \partial_{n} \Lambda_{l p}$ of the gauge field $\Lambda_{l p}$ is parallel to $u_{m}$ with the proportionality coefficient to be $\lambda(x)$ :

$$
\epsilon^{m n l p} \partial_{n} \Lambda_{l p}=\lambda(x) u^{m}
$$


The components of $\Lambda_{l p}$ which do not contribute to (19) are gauged away by the transformations (12). Hence, the only independent field in (19) is $\lambda(x)$. The latter satisfies the condition

$$
\partial_{m}\left(u^{m} \lambda\right)=0
$$

which follows from (19).

It is possible to show that, as in refs. [1, 2], one can eliminate one of the gauge fields (for example $A_{m}^{2}$ ) from the action (9) using its equations of motion and reduce (9) to the ordinary Maxwell action plus terms which contain decoupled auxiliary fields:

$$
S=\int d^{4} x\left(-\frac{1}{4} F_{m n}^{1} F^{1 m n}-\epsilon^{m n p q} \Lambda_{m n} \partial_{p} u_{q}+\lambda(x)\left(u_{m} u^{m}+1\right)\right) .
$$

Thus, we have constructed the manifest Lorentz invariant version of a duality symmetric gauge theory which at the classical level contains in addition to the Maxwell field two decoupled redundant fields $\phi(x)$ (18) and $\lambda(x)$ (19).

Perhaps, one would desire to get rid of this fields, at least at the classical level. A possible way to do this would be to find in the model, or in some its generalization, a hidden symmetry which would allow one to gauge fix $u_{m}(x)$ to a constant vector. The following speculations demonstrate that there is indeed a hint of such a symmetry. The actions (9), (21) are inert under infinitesimal transformations

$$
\begin{gathered}
\delta u_{m}=\partial_{m} \varphi(x) \\
\delta A_{m}^{\alpha}=\varphi \mathcal{L}^{\alpha \beta} \mathcal{F}_{m n}^{\beta} u^{n}, \quad \delta \Lambda_{m n}=\varphi \mathcal{F}_{m}^{\alpha r} u_{r} \mathcal{F}_{n}^{\beta s} u_{s} \mathcal{L}^{\alpha \beta}
\end{gathered}
$$

provided

$$
u^{m} \partial_{m} \varphi=0 .
$$

Eqs. (22) are a localization of the global transformations of the Schwarz-Sen action [1] which substitute off-shell Lorentz invariance in their model (where $\varphi=x^{m} v_{m}$ with $v_{m}$ being a constant velocity vector).

The weak point of the transformations (22) is that the infinitesimal parameter $\varphi(x)$ must be restricted by the condition (23) which is the consequence of (5). As a result of this restriction the r.h.s. of equation (15) is not invariant under (22), while the l.h.s. is invariant. Thus, in general, the equations of motion of the model are not invariant under (22). But if the classical value of $\lambda(x)$ is zero, then the transformations (22) do become a symmetry of the equations of motion (5), (13)-(15) and allow one to gauge fix $u_{m}(x)=\partial_{m} \phi(x)$ (eq.(18)) to be a constant vector with the unit norm (5). At this "critical" point the present model completely coincides with the model of Schwarz and Sen.

But more interesting is to learn what kind of more general (supersymmetric) theory the fields $u_{m}(x)$ and $\Lambda_{m n}(x)$ came from. The natural suggestion is to regard $\Lambda_{m n}(x)$ as an axion potential and $u_{m}(x)$ as a relic of the time-like component of a gravitational 
field vierbein in a model of duality symmetric gauge fields interacting with gravity in such a way that the second and the third term of (9) break the manifest local Lorentz invariance of the tangent space down to $S O(3)$, while the general coordinate invariance of the curved space-time remaines intact. The simple calculus of independent components of the vierbein testifies to this assumption. Indeed, the conventional theory of General Relativity can be formulated equally well in terms of a metric $g_{m n}(x)$ of curved space or in terms of a vierbein $e_{m}^{a}(x)$ (where $a$ is a tangent space index) since the number of independent components of $e_{m}^{a}(x)$ coincides with that of the metric due to the local Lorentz invariance in the tangent space. However, if the local symmetry in the tangent space is smaller than Lorentz symmetry there is a difference in the number of the components of the metric and of the vierbein, and gravity theory formulated in terms of the vierbein will differ from that described by the metric. For example, in our case of the $\mathrm{D}=4$ model, where in the tangent space $\mathrm{SO}(1,3)$ is broken to $\mathrm{SO}(3)$ the vierbein has three more independent components in comparison with the metric. It is just these components of the vierbein which remain when one considers the duality symmetric model in the flat space-time, and these correspond to the three independent components of $u_{m}(x)$.

Of course, all the consequences of this specific local Lorentz symmetry breaking must be understood yet (or, perhaps, there may exist a hidden invariance, as one written in eqs. (22), which substitutes local Lorentz symmetry), but we stress that in the flat limit, due to the presence of $u_{m}(x)$, Lorentz invariance in space-time does take place, and the auxiliary fields decouple, as has been demonstrated above.

With all these points in mind we propose a general coordinate invariant action, which describes the coupling of the duality symmetric gauge fields and a Majorana fermion $\psi^{\mu}(x)$ to gravity, in the following form:

$$
\begin{aligned}
S=\int d^{4} x \operatorname{det}\left(e_{m}^{a}\right) & \left(-\frac{1}{8} F_{m n}^{\alpha} F^{\alpha m n}+\frac{1}{4} e_{(o)}^{m} \mathcal{F}_{m l}^{\alpha} g^{l n} \mathcal{F}_{n p}^{\alpha} e_{(o)}^{p}-\frac{1}{\operatorname{det} e_{m}^{a}} e_{p}^{(o)} \epsilon^{p l m n} \partial_{l} \Lambda_{m n}+R\right) \\
& -i \int d^{4} x\left(\operatorname{det} e_{m}^{a}\right) \bar{\psi}(x) \gamma^{a} e_{a}^{m}\left(\partial_{m}+\frac{1}{4} \omega_{m}^{b c} \gamma_{a} \gamma_{b}\right) \psi(x) .
\end{aligned}
$$

where $g_{m n} \equiv e_{m}^{a} e_{a n}$ is a metric, $R(x)$ is the scalar curvature of the curved space-time and $\omega_{m}^{a b}$ is the $S O(1,3)$ spin connection. When the metric is flat, $e_{m}^{a}$ reduce to the local Lorentz frame fields $u_{m}^{a}=\left(u_{m}, u_{m}^{i}\right.$ ) (eqs. (5), (8) ) and $\omega_{m}^{a b}=u_{n}^{a} \partial_{m} u^{b n}$, the first raw of eq. (24) reduces to (9) and the fermion part becomes the free fermion action

$$
S=-i \int d^{4} x \overline{\hat{\psi}} \gamma^{m} \partial_{m} \hat{\psi}
$$

for a redefined fermion field $\hat{\psi}^{\mu}(x)=v_{\nu}^{\mu}(x) \psi^{\nu}(x)$, where $v_{\nu}^{\mu}(x)$ is a $\operatorname{Spin}(1,3)$ matrix related to $u_{m}^{a}(x)$ through the well known $\operatorname{Spin}(1,3)$-transformation law for the gammamatrices:

$$
v^{-1} \gamma^{m} v=u_{a}^{m} \gamma^{a}
$$


In agreement with a supersymmetric version of [1], the flat limit of the action (24) is supersymmetric under the following transformations of $A_{m}^{\alpha}$ and $\hat{\psi}$ :

$$
\begin{gathered}
\delta A_{m}^{\alpha}=i \overline{\hat{\psi}} \gamma_{m} \epsilon^{\alpha}, \\
\delta \hat{\psi}=\frac{1}{8} F^{\alpha m n} \gamma_{m} \gamma_{n} \epsilon^{\alpha}+\frac{1}{4} u_{p} \mathcal{F}^{\alpha p m} u^{n} \gamma_{m} \gamma_{n} \epsilon^{\alpha},
\end{gathered}
$$

where $\epsilon^{\alpha}$ are constant Majorana spinors subject to the condition $\epsilon^{\alpha}=i \gamma_{5} \mathcal{L}^{\alpha \beta} \epsilon^{\beta}$. On the

mass shell, when $\mathcal{F}_{m n}^{\alpha}=0$ (eq. (17)), the transformations (26) reduce to the standard supersymmetry transformations for a vector supermultiplet $\left(A_{m}^{1}, \hat{\psi}\right)$.

The model considered above admits the generalization to duality symmetric models of antisymmetric gauge fields in various dimensions [1] and may be regarded as a basis for constructing manifestly-covariant duality-symmetric effective actions coupled to supergravity.

Acknowledgements. D.S. is grateful to John Schwarz for discussion on duality, and to Igor Bandos and Dmitrij Volkov for constant interest to this work and fruitful discussion. P.P., D.S. and M.T. would also like to thank Francesco Toppan for illuminative questions.

Work of D.S. was supported in part by the International Science Foundation under the grant N RY 9000, by the State Committee for Science and Technology of Ukraine under the Grant N 2/100 and by the INTAS grants 93-127, 93-493, 93-633.

\section{References}

[1] J. H. Schwarz and A. Sen, Nucl. Phys. B411 (1994) 35.

[2] A. Khoudeir and N. Pantoja, hep-th/9411235.

[3] M. Green and J. H. Schwarz, Phys. Lett. 109B (1982) 444; Phys. Lett. 131B (1984) 367; Nucl. Phys. B243 (1984) 475.

[4] A. Galperin, E. Ivanov, S. Kalitzin, V. Ogievetsky and E. Sokatchev, Class. Quantum Grav. 1 (1984) 498; Class. Quantum Grav. 2 (1985) 155. 